\title{
A Dynamic Replication Scheme of User Plane Data over Lossy Backhaul Links
}

\author{
Yekta Turk $^{\mathrm{a}}$ and Engin Zeydan ${ }^{\mathrm{b}}$ \\ ${ }^{\text {a}}$ Ericsson Research, Istanbul, Turkey, 34396 \\ ${ }^{\mathrm{b}}$ Centre Technologic de Telecomunicacions de Catalunya (CTTC), Castelldefels, Barcelona, Spain, 08860 \\ ${ }^{1}$ Emails: yekta.turk@ericsson.com, engin.zeydan@cttc.cat
}

\begin{abstract}
G networks are characterized by strict latency, jitter and reliability requirements. On the other hand, a lossy backhaul scenario where high packet losses over the backhaul transmission medium can render backhaul links unavailable for service consumption. This necessities a multi-transmission scheme over backhaul using the existing resources of the operators such as base stations and core networks. This can enhance reliability for $5 \mathrm{G}$ backhaul networks to meet the requirements of new 5G services that have strict end-to-end latency requirements. In this paper, we propose a GPRS Tunnelling Protocol User Plane (GTP-U) Protocol Data Unit (PDU) multitransmission scheme that achieves improved Transmission Control Protocol (TCP) latency and packet loss probability at the expense of higher resource (bandwidth) utilization compared to existing traditional systems. The scheme can use several measuring mechanisms such as continuous Two-Way Active Measurement Protocol (TWAMP) testing between core network and base stations, GTP-U user plane echo requests/replies or backhaul quality testing during evolved Packet System Radio Access Bearer (eRAB) establishment phase. This mechanism can also be applied to $\mathrm{X} 2$ interface between base stations to reduce the service latency and improve the backhaul link performance. Our simulation results using ns-3 indicate that by applying the proposed strategy in backhaul systems, the application level packet loss and TCP delay can be improved at the expense of the bandwidth improvement at certain drop or loss levels.

Index Terms-5G, user plane, GTP-U, backhaul, multitransmission.
\end{abstract}

\section{INTRODUCTION}

Time-constraint applications and services can be considered to be the most important feature that are promised with $5 \mathrm{G}$ networks [1]. However, there can be several scenarios where a sudden decrease in the service level of backhaul links in Mobile Network Operator (MNO)'s existing infrastructure can deteriorate the service. These scenarios are predominantly ad-hoc in nature and should be solved using the existing infrastructure facilities in short duration. Therefore, it is highly desirable to be able to create a dynamic self-organized reliable solution to backhaul failures in such conditions. Additionally, existing infrastructure should also be utilized with software modifications that can be integrated to already existing infrastructure. Otherwise new deployments of network elements need to be followed.

GPRS Tunneling Protocol (GTP) protocol is specific tunneling protocol used between end nodes in General Packet Radio Service (GPRS) backbone network and is a group of Internet Protocol (IP)-based communications protocols used to carry general packet radio service. It includes both signaling and user data transfer procedures in interfaces such as S1-U, S10, S11, S4, S5/S8 and S3 in cellular network infrastructure. Generally GPRS Tunneling Protocol Control Plane (GTP-C) and GPRS Tunneling Protocol User Plane (GTP-U) protocols are used for control and user planes of Packet Switched (PS) traffic. Each user plane connection requires GTP-U tunnel across the Evolved Packet System (EPS) backbone network to transmit the user payload. Hence, GTP-U is used to transfer the user data in separated tunnels for each Packet Data Protocol (PDP) context. GTP$\mathrm{U}$ can be seen as a framing protocol which allows multiprotocol packets to be tunneled through the EPS backbone to provide a service for carrying user data packets. On the other hand, GTP-C tunnel, which is the control section of the GTP standard, is used to create, modify and delete tunnels.

An example for the end-to-end latency requirements of $5 \mathrm{G}$ services for vertical industries is summarized in Table I as described in [2]. In an end-to-end access network, new generation $5 \mathrm{G}$ networks should be provided with very fast and critical communication to meet the stringent numerical requirements outlined in Table I. Moreover, this fast transmission should not only be available in the radio interface, but in the backhaul interface as well. Improvements on NGUU interface are not adequate to improve the end-to-end latency of communication systems in cases where backhaul network becomes the bottleneck of communication. The slightest error in backhaul networks can yield inefficiency

TABLE I

3GPPP DEFINEd LATENCY NEEDS For VeRTiCAL INDUSTRIES [2]

\begin{tabular}{r|l}
\hline Service & E2E Latency Requirements \\
\hline Live Streaming & $<20 \mathrm{~ms}$ \\
\hline Smart Grid & $<50 \mathrm{~ms}$ \\
\hline Time-critical sensing & $<30 \mathrm{~ms}$ \\
\hline $\begin{array}{r}\text { Real-time control } \\
\text { for discrete automation }\end{array}$ & $\leq 1 \mathrm{~ms}$ \\
\hline $\begin{array}{r}\text { Remote drone operation \& } \\
\text { Cooperative farm machinery }\end{array}$ & $\leq 30 \mathrm{~ms}$ \\
\hline Real-time video & $<100 \mathrm{~ms}$ \\
\hline
\end{tabular}




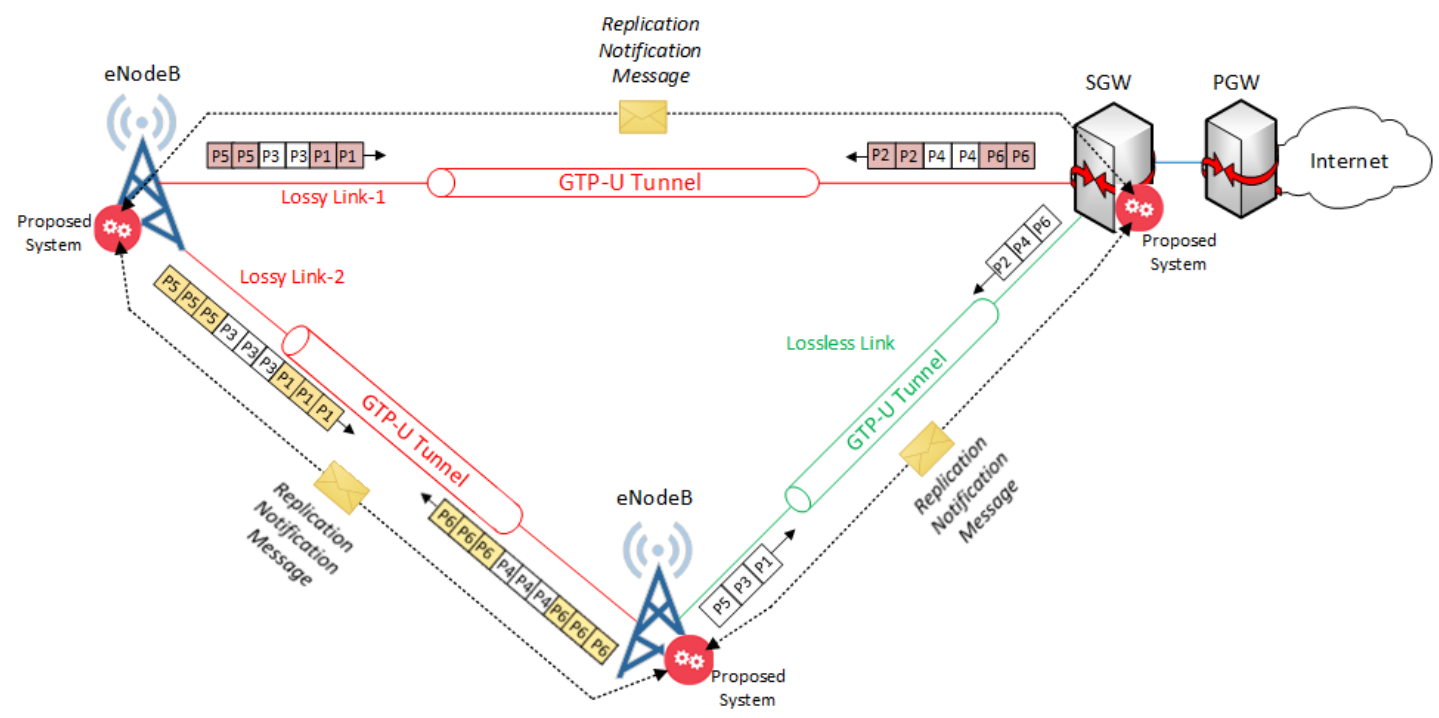

Fig. 1: Operation of the proposed system (marked in red as a feature) in links that have different levels of packet loss rate and in a lossless link.

of the specified $5 \mathrm{G}$ services. For this reason, additional measures need to be taken between the core network and $5 \mathrm{G}$ base station to mitigate the potential delay that may occur over the backhaul link. Therefore, an integrated access and backhaul approach is necessary to keep the end-to-end Quality-of-Service (QoS) requirements for $5 \mathrm{G}$ services.

In this paper, our scenario predominantly applies for $5 \mathrm{G}$ services in which they have to meet the new challenges such as severe resource constraints, unreliable communication links as well as other issues including limited computational capability and scalability problems. Our analysis is based on the fact that with the integrated access and backhaul networks, a better network providing services with higher reliability and lower latency can be delivered. For this reason, we follow an additional replication strategy over backhaul network that is complementary to proactive decisions taken at the radio access level.

\section{A. Related Works}

Replication strategies that enhance the capabilities of network elements have been studied quite a lot in the literature [3]. However, the majority of proposals concentrate on single access network side such as Transmission Time Interval (TTI) bundling or Radio link control (RLC) segmentation for edge users to improve the reliability in radio side. These can be useful for appropriate applications of radio quality enhancing features such as Carrier Aggregation (CA), Coordinated Multipoint (CoMP) [4] or Single Frequency Network (SFN) [5]. The effect of bundling or multiplexing over the air interface for data or/and control transmission is well studied in the literature [6], [7]. However, enhancing multi-transmission capability in backhaul networks for GTP tunneling is still an under-investigated topic. In [8], authors emulated the effect of backhaul packet loss on the S1$U$ interface and its impact on performance of end-user applications. An integrated fronthaul/backhaul transport network forwarding method in a software-controlled network to adapt the capacity demand is studied in [9]. In [10], authors proposed to use bundling and multiplexing for the voice packets sent to a mobile backhaul.

In standardization works related to GTP, 3GPP reference [11] states that Packet Data Gateway (PGW) supports sending sequence number in G-PDUs. Serving Gateway (SGW) supports relaying sequence number for the indirect data forwarding when sequence numbers are added by PGW. On the other hand, the need for the replacement of GTP protocol in mobile network is under consideration and studied by Internet Engineering Task Force (IETF) and suitable protocols for user plane in next-generation networks are evaluated in [12]. In standard GTP-U operation, if multiple copies of the same Protocol Data Unit (PDU) with same sequence number are received at the receiver side (either at SGW or eNodeB), only one of those PDUs are transmitted to upper layers while the rest are discarded. Under normal circumstances, sequence numbering is only over GTP-C PDUs. However, 3rd Generation Partnership Project (3GPP) only advises on using sequence numbering in GTP-U in case it is required by MNOs. Assigning sequence numbers to the GTP-U path management messages, such as echo request and reply messages is mandatory but is not needed for other GTP-U messages as defined in [11].

\section{B. Our Contributions}

Dynamic multi-transmission in backhaul based on measurements can help to bring additional layer of reliability to $5 \mathrm{G}$ services. In this paper, we study a method without disturbing the existing features of the standard. Our method 
exploits the advantages of multi-transmitting of PDUs, and the optional field of sequence numbering for GTP-U PDUs. Our main contributions can be summarized as follows: (i) We study a system that protects $5 \mathrm{G}$ services against the backhaul network failures by periodically collecting network drop statistics and executing multi-transmission of GTP-U PDUs. (ii) We analyze of trade-off between the decrease in packet loss probability and application level Transmission Control Protocol (TCP) latency and increase in utilized bandwidth needs of multiple transmission technique of GTP-U PDUs in backhaul via ns-3 simulation analysis.

The rest of the paper is organized as follows: System model and concepts as well as general architecture of the proposed system is given in Section II. Section III is describing the details of the proposed backhaul transmission scheme in mobile networks. In Section IV, simulation results of the considered scheme are provided. Finally, we provide conclusions and future work in Section V.

\section{System Model AND CONCEPTS}

\section{A. Design Principles}

For any user equipment (UE), a drop or poor quality on the transport network can be inferred from signalling traffic when EPS Radio Access Bearer (eRAB) is setup between eNodeB and core network. Additionally, a dynamic two-way active measurement protocol (TWAMP) client-server [13] test can be run continuously from eNodeB (or gNodeB) to core network or from core network to the eNodeB(gNodeB). GTP-U user plane echo can also be used test the quality of the transmission. The proposed dynamic multitransmission capability can be installed between eNodeB and packet core network where the GTP-U tunnel is present and can also be used in other interfaces such as in X2-U interface where the GTP-U tunnel is present as given in Fig. 1. After a failure condition is detected over the backhaul link, the multiple transmission feature is activated and GTPU PDUs are replicated over the lossy backhaul network. The multi-transmission level can be increased or decreased depending on the loss rate in backhaul network and the target reliability value until reaching an achievable level. By this way, the network can react to the instantaneous network failures and the service continuity can be enabled over the unreliable link.

In our network, let $\mathbf{G}$ be the total number of GTP-PDUs in backhaul, $\mathbf{D}$ be the number of dropped GTP-PDUs on the backhaul link due to packet loss, $\mathbf{S}$ be the number of successfully received GTP-PDUs at a given instant. Assume that $\mathbf{R}$ denotes the link data rate (bits/second), $\mathbf{P}$ is Packet size (bits), $\mathbf{L}$ is the link distance (meters), $\mathbf{N}$ to be node processing delay (seconds), $\mathbf{Q}$ to be Queue depth (bits) and $\rho$ to be propagation speed of medium (e.g. speed in fiber is $3 \times 10^{6}$ (meters/second)). Then, the drop rate probability of GTP-PDUs $p_{D R}$ is calculated as

$$
p_{D R}=\frac{\mathbf{D}}{\mathbf{G}},
$$

and the GTP-PDU packet loss probability $p_{P L}$, is defined as

$$
p_{P L}=1-\frac{\mathbf{S}}{\mathbf{G}} \text {. }
$$

Finally, application level delay, $\kappa$ is calculated as

$$
\begin{aligned}
\kappa= & \frac{\mathbf{P}}{\mathbf{R}}(\text { Frame Serialization Time }) \\
& +\frac{\mathbf{L}}{\rho}(\text { Link Media Delay }) \\
& +\frac{\mathbf{Q}}{R}(\text { Queueing Delay })+\mathbf{N} .
\end{aligned}
$$

\section{B. Faulty Backhaul Problem}

Unreliable backhaul communication links, together with low latency requirements of $5 \mathrm{G}$ services hampers with QoS and can often lead to unstability in networks. Next generation 5G networks are envisioned to be SoftwareDefined Networking (SDN)-based and network automation will be a key feature of building a zero touch network infrastructure [14]. However, physical devices will already be on the physical sites. Therefore, in real-life applications, transmission structure such as the star topology from any to any point transmission will not be provided by MNOs due to Capital Expenditure (CaPEX) and Operational Expenditure (OpEX) issues. This means that there may always be cases where error and packet loss are possible. Considering that there may be many users connected to a base station, a problem with backhaul will have a negative impact on many users' performance. At the same time, there may be several causes to trigger replication of GTP-U PDUs in backhaul. Some of the problems in backhaul links that can cause packet loss and user Quality-of-Experience (QoE) degradation can be summarized as follows: (i) Packet loss in backhaul: This problem is usually caused by the underlying impairments in the physical systems. For example, the weakening of fiber cables on transmission lines, problems in the connected Small form-factor pluggable transceivers (SFPs), the power supply problems of the equipment such as the Dense Wavelength Division Multiplexing (DWDM), Synchronous Optical Networking (SDH), IP/Multiprotocol Label Switching (MPLS) routers, which is the source of the fiber, or the presence of Radio Link (R/L) equipment in the carrier network. Additional problems such as the failure of proper supply of Line of Sight (LOS) links may initiate the loss of packets in the network links. ii) Burst errors: These fault types occur with a certain time interval and cause the packets in that range to be lost. These may be periodic or may occur at different times. The burst errors in the backhaul links are similar to the packet loss for UEs. Since PDU cannot be extracted appropriately, it will be retransmitted in TCP-based communication. In the case of User Datagram Protocol (UDP)-based communication, it will have detrimental affect on UE. iii) Link saturation: Another condition that may cause loss is due to the saturation of the links. The backbone carrier line between 


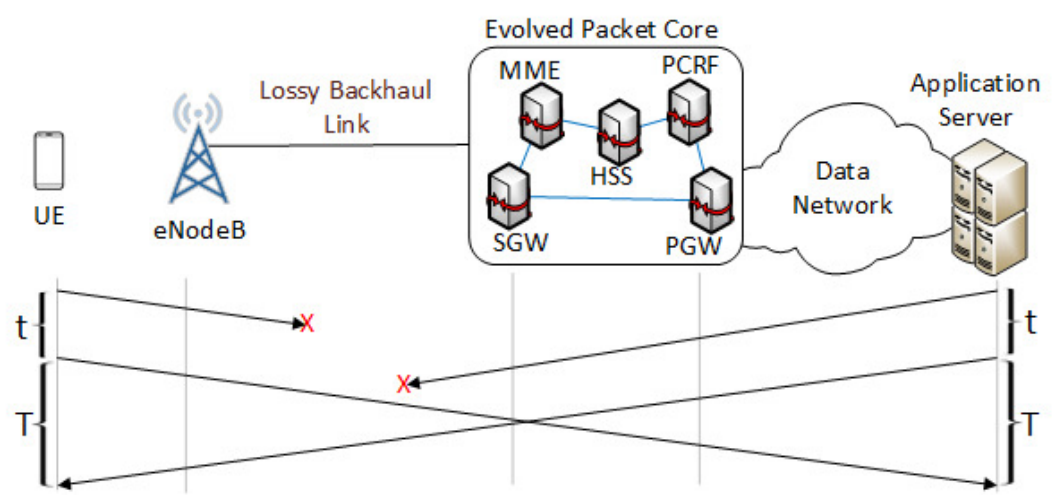

(a)

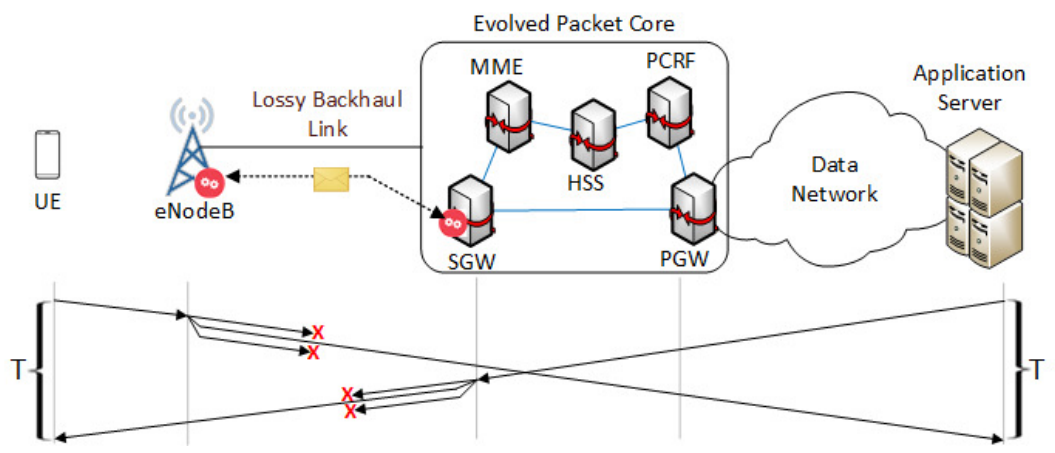

(b)

Fig. 2: a) Standard GTP-U operation b) Proposed GTP-U multi-transmission operation.

eNodeB and EPS can serve multiple UEs simultaneously. For example, if there is a virtual leased circuit leased over a fixed network, there may be other corporate circuits and broadband services belonging to the fixed operator. If the saturation status occurs in the main carrier network of the fixed operator, some of the traffic that may be lost can be recovered later by activating multiple transmissions.

\section{Proposed Backhaul Transmission in Mobile NETWORKS}

\section{A. Dynamic Measurements of Backhaul Links}

To implement a dynamic and resilient backhaul design, we suggest a multi-transmission scheme as detailed below. For measuring the backhaul link performance between eNodeB and SGW in core network, each network element building a GTP tunnel is equipped with additional measurement capabilities similar to client-server architecture as described in Section II-A. Each network element maintains a network link status that keeps track of the current level of backhaul link quality in either Download (DL) or Upload (UL) direction. Further, depending on the link quality a multiplication factor, $\alpha$ is established. The multiplication factor can depend on many resource components including power consumption, energy, memory of network elements and available bandwidth in backhaul. The network elements participating GTP tunnel creation process monitor the link and generate a replication of GTP-U PDUs in case

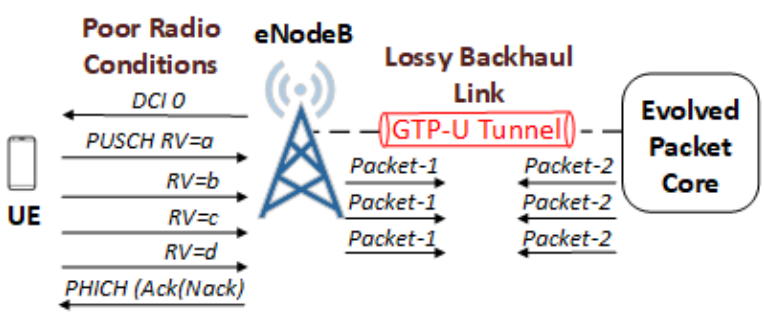

Fig. 3: TTI Bundling in Radio Network and Proposed System in Backhaul Network.

the link drop rate percentage increases. Replication can also be triggered in case the service response time and/or completion time approach to QoS requirement limits. This may occur due to one of the three cases mentioned in Section II-B.

\section{B. Dynamic Multi-Transmission of GTP-U PDUs}

Fig 1 shows an illustration of operation of the proposed system in links that have different levels of packet loss rates and that are in lossless link. Depending on the characteristics of the lossy link which is obtained via above measurement methodology, multiplication factor can be adjusted by eNodeBs and SGW. For example in Fig 1, lossy link-1 and lossy link-2 exhibit different link characteristics where the loss on lossy link-2 is more severe than lossy 


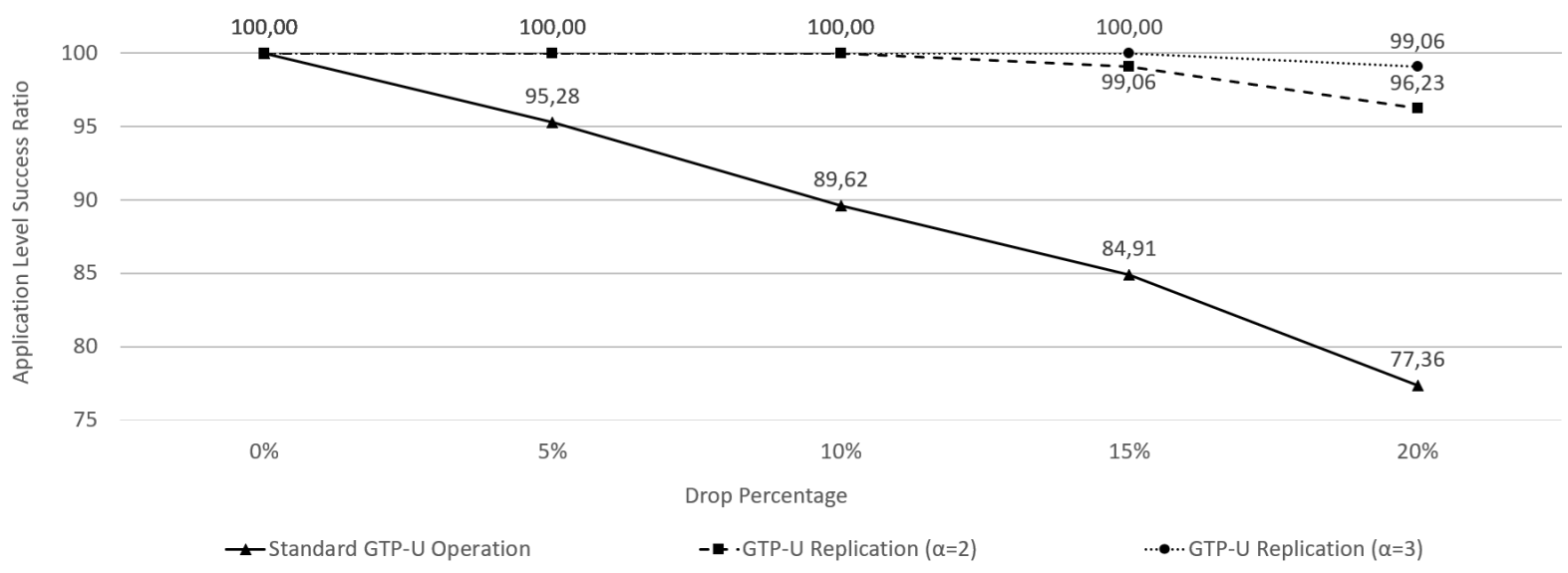

Fig. 4: Application level packet success rate versus drop rate performances of standard GTP-U operation and different multiplication factors $\alpha$ of GTP-U multi-transmission

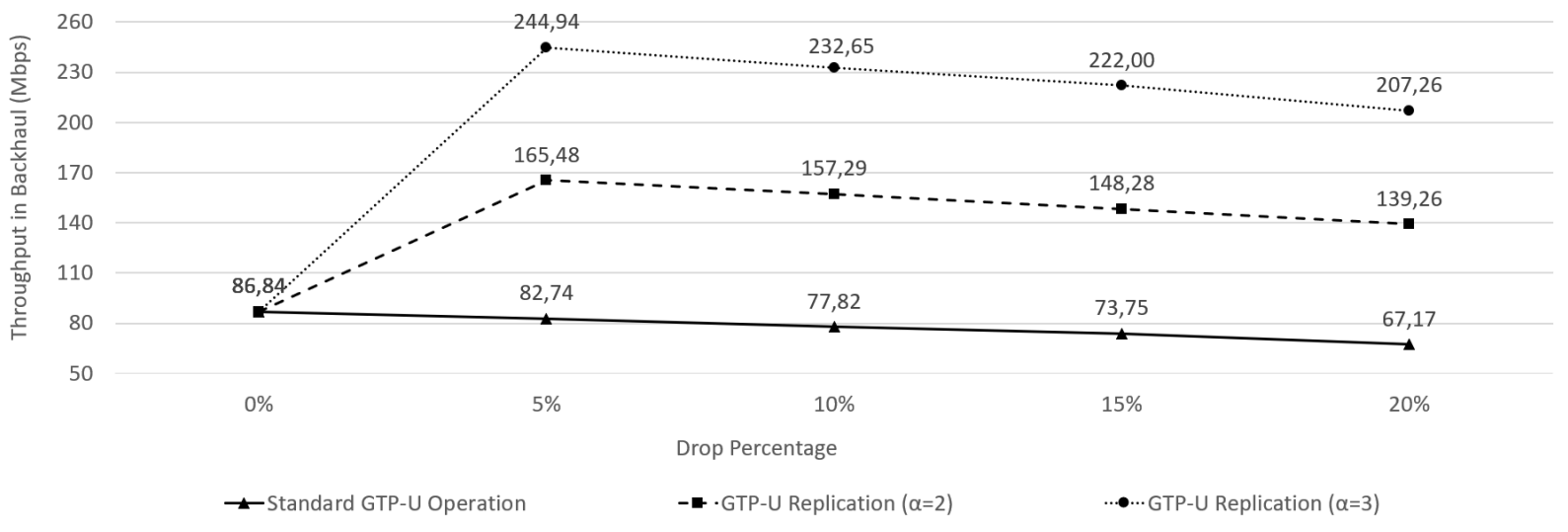

Fig. 5: Bandwidth throughput versus drop rate of standard GTP-U operation and different multiplication factors $\alpha$ of GTP-U multi-transmission

link-1. Then, the amount of replicated PDUs over this channel is selected to be higher to increase the probability of reception.

The operation structure of standard GTP-U operation and our proposed GTP-U multi-transmission methodology over a lossy link for TCP based end user communication are given in Fig. 2. In the proposed architecture, sequence numbering on SGW and eNodeB must be activated to drop GTP-U PDUs that are transmitted and received multiple times. Standart GTP-U operation given in Fig. 2a does not have any multi-transmission structure, hence if GTP$\mathrm{U}$ PDU is lost on the backhaul link after $t$ amount of time, a new transmission takes place and hence the total transmission time of one GTP-U PDU can be calculated as $t+T$ assuming a successful transmission takes $T$ units of time. On the other hand, together with the proposed GTP$\mathrm{U}$ multi-transmission methodology as given in Fig. 2b, even if some of PDUs are lost on the lossy channel, due to higher chances of success probability of the transmission time of one GTP-U PDU remains at $T$.

\section{Comparison with TTI bundling}

Fig. 3 shows TTI bundling in radio network as well as the proposed system in backhaul network. TTI bundling on the radio network can be considered as an analogy to the proposed system. TTI bundling is introduced in uplink of Frequency Division Duplex (FDD) and Time Division Duplex (TDD) based Long Term Evolution (LTE) systems to increase the coverage. In TTI bundling, a transport block is sent multiple times in consecutive frames without waiting for Hybrid Automatic Repeat Request (HARQ) Acknowledgment (ACK)/Negative Acknowledgment (NACK) messages in bad radio channel conditions. The motivations are to use low power transmission at UEs and ensure delay sensitive traffic 


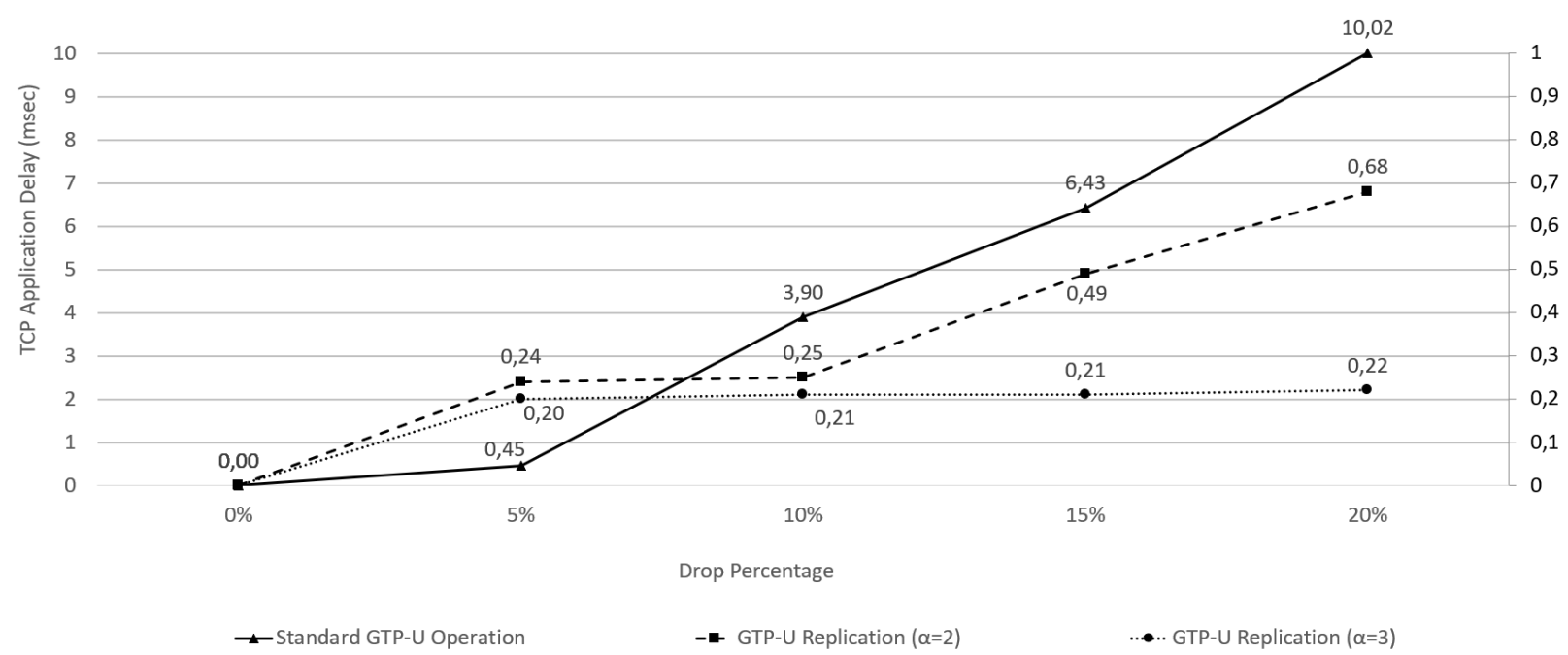

Fig. 6: End user's TCP application delay versus drop rate of standard GTP-U operation and different multiplication factors $\alpha$ of GTP-U multi-transmission.

such as voice over IP (VoIP) transmission over the highlatency links. The main aim of TTI bundling is to increase the probabilities of reception and successful decoding at the receiver with low latency. It has the potential to increase the link budget up to $4 \mathrm{~dB}$ [15].

There exists a similarity between the concept of TTI bundling in radio access networks and the proposed GTP$\mathrm{U}$ multi-transmission scheme in backhaul networks. While the purposes of them are different, the idea of sending multiple copies of the PDUs in a row remains the same. The main difference is that in TTI bundling, a common low layer header information is shared across the TTI bundle. Similarly, dynamic multiple transmission can also be activated due to the experienced delay in the backhaul link for the next generation networks. The disadvantages of these schemes are that with TTI bundling spectrum efficiency is decreased, while with the proposed system high bandwidth usage may be occur in backhaul link.

\section{Simulation Results And Discussion}

In this section, we used LENA LTE-EPC module of [16] which is based on ns-3 and C++ coding to emulate the network. The LTE ns3 module enables us to simulate the proposed system in an end-to-end cellular network with a complete implementation of TCP/IP stack. Our evaluation results are validating the gains as well as the trade-offs involved in GTP-U multi-transmission scheme. Table II summarizes the simulation parameters that are used in our evaluations. We assume no congestion in links and no processing delay, i.e. $\mathbf{Q}=\mathbf{N}=0$.

We compare the performance of our proposed multitransmission of GTP-U PDUs introduced in Section III with standard GTP-U transmission scheme along with different number of replications. Fig 4 shows the packet loss performance comparisons of standard GTP-U operation
TABLE II Simulation PARAMETERS

\begin{tabular}{r|l}
\hline Parameters & Value \\
\hline Test Duration & $10 \mathrm{~s}$ \\
\hline Number of UEs & 10 \\
\hline Packet size & 1024 bytes \\
\hline Inter-packet interval & $10 \mathrm{~ms}$ \\
\hline S1 Transport Bandwidth & $1000 \mathrm{Mbps}$ \\
\hline Carrier Frequency & $1800 \mathrm{Mhz}$ \\
\hline Bandwidth & $20 \mathrm{Mhz}$ \\
\hline UGPP Channal Scenario & Urban \\
\hline UAC Scheduler & Proportional Fair $(\mathrm{PF})$ \\
\hline Subframe duration & $1 \mathrm{~ms}$ \\
\hline RLC buffer size for UEs & $1 \mathrm{~ms}$ \\
\hline eNodeB Power & $46 \mathrm{dBm}$ \\
\hline Noise Power Spectral Density & $-174 \mathrm{dBm} / \mathrm{Hz}$ \\
\hline Antenna Configuration & $1 \times 1$ \\
\hline Number of RBs & 100 \\
\hline Path Loss Model & $128.1+37.6 \log { }_{10} d$ [dB] \\
\hline Sumber of Resolvable Path & 7 (complex-Gaussian) \\
\hline Shadowing & Log-Normal (mean: 0, s.d.: $6 \mathrm{~dB})$ \\
\hline &
\end{tabular}

and the utilized GTP-U multi-transmission with different multiplication factors, $\alpha$. When the drop rate percentage is zero, the proposed system acts as the standard operation, hence the application level success ratios are the same. Note that we assume an ideal backhaul where S1 transmit delay is selected to be zero. We observe that as the drop percentage in backhaul link increases, application level packet success ratio decreases. However, standard GTPU operations' success rate experiences dramatic decrease, where its success rate can decrease up to $77.36 \%$ when the drop rate goes down to $20 \%$ levels. On the other hand, the proposed scheme can derive benefit from replication of GTP-U traffic between SGW and eNodeB nodes. Due to the incorporation of multi-transmission in the proposed 
scheme, the drop percentage is reduced as GTP-U PDU is highly likely to be successfully received within the specific nodes. For example together with GTP-U multiplication factor $\alpha=2$, the success ratio is increased to $96.23 \%$ and with $\alpha=3$, it has increased to $99.06 \%$ when the drop rate is around $20 \%$. Hence, the gain in drop rate is primarily contributed by the increased reliability of cellular backhaul link.

In Fig. 5, we plot the bandwidth increase against the drop rate. The figure shows the amount of traffic only over the backhaul, not between eNodeB and UE, and UEs are not aware of the generated multiple traffic by the system. First of all, each of schemes suffer from higher drop rate. When the drop rate percentage is zero, the proposed system acts as the standard operation, hence the utilized backhaul bandwidths are the same. It is evident that multitransmission requires more bandwidth compared to normal GTP-U operation. It is observed that the bandwidth utilization in both the levels of transmission scheme is higher compared to standard operation. Due to higher number of replicas for increasing the success ratio and consequently, the resource utilization in the system also increases. Compared to normal operation, the bandwidth utilization has increased to $139.26 \mathrm{Mbps}$ for GTP-U multiplication factor $\alpha=2$ and to $207.7 \mathrm{Mbps}$ for GTP-U multiplication factor $\alpha=3$ when the drop rate approaches $20 \%$. Note also that as the drop rate increases, the bandwidth utilization of each transmission scheme decreases due to lower number of success rate of reception.

Fig. 6 shows the delay values of UE's TCP application versus increasing drop rate. Similar to before analysis, this figure has shown the comparisons between the standard GTP-U operation and different multiplication factors $\alpha$ of GTP-U multi-transmission. When there is no drop rate, the TCP delay starts at zero based on our assumptions. As the drop rate increases, the TCP delay increases the most in standard GTP-U operation due to increased chances of drop rates and re-transmissions as also shown in Fig. 2. As multitransmission opportunity arises, TCP delay is reduced. For example, at $20 \%$ drop rate, the TCP delay with GTP-U multiplication factor $\alpha=2$ is $0.68 \mathrm{msec}$ whereas it drops to 0.22 msec when GTP-U multiplication factor becomes $\alpha=3$. On the other hand, standard GTP-U operation experiences a high delay value of $10.02 \mathrm{msec}$ at $20 \%$ drop rate.

\section{Conclusions And Future Work}

In this paper, we propose a GTP-U replication scheme targeted for increasing the reliability of backhaul networks for service oriented design. The proposed scheme makes use of multi-transmission of GTP-U PDUs along interfaces where GTP tunneling are utilized such as X2 and S1 interfaces. Through ns-3 simulations, we analyzed tradeoff between the packet success rate, TCP-level delay and bandwidth utilization demands of using multiple GTP-U transmission strategy. Our results indicate the success rate as well as TCP delay can improve at the expense of higher bandwidth requirements at certain drop rate percentages with multi-transmission of GTP-U PDUs. As a future work, priority user traffic can only be multi-transmitted with improvements on both eNodeB and SGW sides. At the same time, Deep Package Inspection (DPI) and machine learning methods can also be integrated in the system for traffic inspection and multiple transmission decision.

\section{ACKNOWLEDGEMENTS}

This work was partially funded by Spanish MINECO grant TEC2017-88373-R (5G-REFINE) and by Generalitat de Catalunya grant 2017 SGR 1195 and partially supported by The Scientific and Technological Research Council of Turkey in part under 1515 Frontier R\&D Laboratories Support Program with project no: 5169902.

\section{REFERENCES}

[1] O. Narmanlioglu, E. Zeydan, and S. S. Arslan, "Service-aware multiresource allocation in software-defined next generation cellular networks,” IEEE Access, vol. 6, pp. 20348-20363, 2018.

[2] 3GPP Technical Specification, "Service requirements for the $5 \mathrm{~g}$ system,” 3GPP TS 22.261 V16.6.0, 2018.

[3] E. Spaho, L. Barolli, and F. Xhafa, "Data replication strategies in p2p systems: A survey," in 2014 17th International Conference on NetworkBased Information Systems, pp. 302-309, IEEE, 2014.

[4] Y. Turk, E. Zeydan, and C. A. Akbulut, "Experimental performance evaluations of comp and ca in centralized radio access networks," Telecommunication Systems, pp. 1-16, 2019.

[5] Y. Turk, E. Zeydan, and C. A. Akbulut, "On performance analysis of single frequency network with c-ran,” IEEE Access, vol. 7, pp. 15021519, 2019.

[6] C. P. Li, C. Li, J. Jiang, W. Chen, H. Xu, and H. Sun, “Tti bundling for urllc ul/dl transmissions," Apr. 12 2018. US Patent App. 15/480,019.

[7] C. Ramesh and V. Karthik, "A novel adaptive tti bundling scheme in lte system," in 2017 IEEE International Conference on Advanced Networks and Telecommunications Systems (ANTS), pp. 1-4, IEEE, 2017.

[8] A. Rusan and R. Vasiu, "Emulation of backhaul packet loss on the lte sl-u interface and impact on end user throughput," in IEEE International Conference on Intelligent Computer Communication and Processing (ICCP), pp. 529-536, IEEE, 2015.

[9] T. Deiß et al., "Packet forwarding for heterogeneous technologies for integrated fronthaul/backhaul," in European Conference on Networks and Communications (EuCNC), pp. 133-137, IEEE, 2016.

[10] Á. Drozdy, Z. Vincze, and C. Vulkán, "Bundling and multiplexing in packet based mobile backhaul," in 2010 European Wireless Conference (EW), pp. 346-353, IEEE, 2010.

[11] 3GPP Technical Specification, "General packet radio system (gprs) tunnelling protocol user plane (gtpvl-u)," 3GPP TS 29.281 V15.5.0, 2018.

[12] K. Bogineni, A. Akhavain, T. Herbert, D. Farinacci, and A. RodriguezNatal, "Optimized mobile user plane solutions for $5 \mathrm{~g}$," draft-boginenidmm-optimized-mobile-user-plane-00, 2018.

[13] K. Hedayat, R. Krzanowski, A. Morton, K. Yum, and J. Babiarz, "A two-way active measurement protocol," in IETF RFC 5357, 2008.

[14] ETSI, “ZSM ISG information.” https://portal.etsi.org/zsm, 2019. [Online; accessed 24-February-2019].

[15] R. Susitaival and M. Meyer, "Lte coverage improvement by tti bundling," in VTC Spring 2009-IEEE 69th Vehicular Technology Conference, pp. 1-5, IEEE, 2009.

[16] N. Baldo, M. Miozzo, M. Requena-Esteso, and J. Nin-Guerrero, "An open source product-oriented lte network simulator based on ns-3," in Proceedings of the 14th ACM international conference on Modeling, analysis and simulation of wireless and mobile systems, pp. 293-298, ACM, 2011. 